\title{
Factores asociados al cumplimiento de la normatividad de gestión de residuos municipales de Juliaca, Perú
}

\author{
Factors associated with compliance with municipal waste management \\ regulations in Juliaca, Peru \\ Madona Tito-Betancur, ${ }^{1}$ Marisol Huamán Espejo, ${ }^{2}$ Oscar Mamani-Benito ${ }^{3 a}$ \\ Universidad Tecnológica del Perú, Arequipa, Perú ${ }^{1}$ \\ Universidad Peruana Unión, Lima, Perú ${ }^{2}$ \\ Universidad Peruana Unión, Juliaca, Perú ${ }^{3}$ \\ (iD) ORCID ID: https://orcid.org/0000-0002-4611-6899 ${ }^{1}$ \\ (iD) ORDID ID: https://orcid.org/0000-0002-1590-3339² \\ (iD) ORCID ID: https://orcid.org/0000-0002-9818-2601 $^{3}$
}

Recibido:22 de marzo de 2021

Aceptado: 30 de junio de 2021

\section{Resumen}

La gestión de residuos municipales es un problema critico en el Perú, por ello, se tuvo el objetivo de determinar los factores asociados que explican el cumplimiento de la normatividad de la gestión Integral de Residuos Sólidos en la municipalidad de San Román. Corresponde a un estudio explicativo y transversal, donde la población estuvo conformada por 96 trabajadores administrativos y operativos del área de gestión ambiental, a quienes se le aplicó una escala de cumplimiento de la Gestión Integral de residuos Sólidos y una escala de factores asociados. Según el análisis de regresión el factor gestión del presupuesto (administrativos: $\mathrm{R} 2=.258, \beta=.531, \mathrm{p}<$ .01 ; operativos: $\mathrm{R} 2=.249, \beta=.420, \mathrm{p}<.01$ ), el factor gestión municipal (administrativos: $\mathrm{R} 2=$ $.598, \beta=.782, \mathrm{p}<.01$; operativos: $\mathrm{R} 2=.258, \beta=.420, \mathrm{p}<.01$ ) y el factor responsabilidad social (administrativos: $\mathrm{R} 2=.592, \beta=.778, \mathrm{p}<.01$; operativos: $\mathrm{R} 2=.165, \beta=.422, \mathrm{p}<.01$ ), explican significativamente el cumplimiento de la normatividad de gestión de residuos municipales. Se concluye que la gestión del presupuesto, la gestión municipal y la responsabilidad social, son factores que explican el cumplimiento de la normatividad de gestión residuos en la municipalidad de Juliaca. Esto debe tener repercusiones a nivel administrativo en la gestión municipal actual.

Palabras clave: auditoria de gestión, presupuesto del estado, gestión ambiental, responsabilidad social, residuos sólidos, Juliaca 


\begin{abstract}
Municipal waste management is a critical problem in Peru, therefore, the objective was to determine the associated factors that explain compliance with the regulations of the Integrated Solid Waste Management in the municipality of San Roman. It corresponds to an explanatory and cross-sectional study, where the population consisted of 96 administrative and operational workers of the environmental management area, to whom a scale of compliance with the Integrated Solid Waste Management and a scale of associated factors were applied. According to the regression analysis, the budget management factor (administrative: $\mathrm{R} 2=.258, \beta=.531, \mathrm{p}<.01$; operatives: $\mathrm{R} 2=.249, \beta=.420, \mathrm{p}<.01$ ), the municipal management factor (administrative: $\mathrm{R} 2=.598, \beta=$ $.782, \mathrm{p}<.01$; operatives: $\mathrm{R} 2=.258, \beta=.420, \mathrm{p}<.01$ ) and the social responsibility factor (administrative: $\mathrm{R} 2=.592, \beta=.778, \mathrm{p}<.01$; operational: $\mathrm{R} 2=.165, \beta=.422, \mathrm{p}<.01$ ), significantly explain compliance with municipal waste management regulations. It is concluded that budget management, municipal management and social responsibility are factors that explain compliance with waste management regulations in the municipality of Juliaca. This should have repercussions at the administrative level in the current municipal management.
\end{abstract}

Keywords: performance audit, state budget, environmental management, social responsibility, social responsibility, solid waste, Juliaca

\title{
Introducción
}

El problema de la gestión de residuos sólidos es preocupación en todo gobierno a nivel nacional, regional y local (Quillos et al., 2018). Toda vez que la población mundial se ha venido incrementando con el devenir del tiempo, han ocurrido cambios en el estilo de vida de la población que han conllevado a la generación de residuos, así como la preocupación por su manejo (Abarca et al., 2018).

En el año 2018 se generaron cerca de 2010 millones de toneladas de residuos sólidos municipales en el mundo, de esta cantidad por lo menos $33 \%$ no se gestionaron, lo cual aumentó el riesgo para el medio ambiente (Banco Mundial, 2018). Según el portal Infobae (2017) China es el país que genera mayor cantidad de residuos sólidos (300 millones de toneladas), seguido por Estados Unidos con 228 millones de toneladas, a la par de la India con también 226 millones de toneladas. En Latinoamérica, Brasil aparece en dicho ranking, evidenciando 62 millones de toneladas de residuos por año.

En cuanto al Perú, se calcula que en la actualidad se generan aproximadamente 19 mil toneladas de residuos sólidos municipales. Aproximadamente cerca del $50 \%$ de estos se producen en Lima Metropolitana y la provincia constitucional del Callao. Por otra parte, del total de residuos que se generan, aproximadamente $54 \%$ corresponde a residuos sólidos orgánicos y $20 \%$ a residuos 
sólidos inorgánicos valorizables. Ahora, de este grupo el 52\% son dispuestos en rellenos sanitarios, mientras que el resto se vierte en botaderos informales y espacios no autorizados que ponen en riesgo la calidad del ambiente y la salud de las personas (Ministerio de Economia y Finanzas, 2019).

En este contexto, la literatura da cuenta que el tratamiento y eliminación de residuos ha sido un terreno exclusivamente prioritario en países donde el ingreso es alto o moderado, y, por tanto su gestión se lleva con mejores resultados. En cambio, en los países con ingresos a nivel bajo, cerca del 93\% de residuos se quema o tira en carreteras, campos abiertos o canales fluviales, teniendo resultados desfavorables para el medio ambiente (Salazar, 2020).

A pesar de que los países en Latinoamérica han instaurado normas para la adecuada gestión de residuos sólidos (Sánchez-Muñoz et al., 2019), desde hace muchos años es notorio el fenómeno relacionado al incumplimiento de la normatividad por parte de los gobiernos municipales, sobre todo en el Perú, país donde este problema tiene una larga data y antigüedad (Bustios et al., 2013). Por ejemplo, respecto a la Ley de Gestión Integral de Residuos Sólidos, ya desde el año 2014 el Organismo de Evaluación y Fiscalización Ambiental (OEFA) informaba que solo 17 gobiernos regionales habían presentado su Plan Anual de Evaluación y Fiscalización Ambiental (PLANEFA), documento clave para priorizar acciones que prevengan impactos severos al ambiente (Diario Gestión, 2014).

Ante esta problemática, urge la necesidad de investigar cuales son los factores que explican el incumplimiento de las normas para la gestión de residuos en el Perú, en este caso, referida a la Ley de Gestión Integral de Residuos Sólidos aprobada mediante el decreto legislativo $\mathrm{N}^{\circ} 1278$ en el año 2017 (Diario Oficial El Peruano, 2017). Al respecto, la literatura científica da cuenta de estudios donde destaca la gestión del presupuesto, la gestión pública y la responsabilidad social (Abelino-Torres et al., 2019; Tumi \& Escobar-Mamani, 2018).

Respecto a la gestión presupuestal, esta hace referencia a la expresión cuantitativa, económicamente hablando, de lo que se dispone y que se necesita gestionar para el manejo de residuos sólidos a cargo de la administración municipal vigente (Oldenhage, 2016). Aunado a esto, se sabe que una de las fuentes de financiamiento es el Ministerio de Economía y Finanzas (MEF) a través de la estrategia Presupuesto por Resultados $(\mathrm{PpR})$, creada para asegurar la calidad y capacidad del gasto público tomando como directriz el programa presupuestal $\mathrm{N}^{\circ}$ 0036: gestión integral de residuos sólidos (MEF, 2020). 
Seguidamente, en cuanto a la gestión pública, tiene que ver con el conjunto de procesos y acciones a nivel administrativo con los cuales los gobiernos locales deben gestionar los residuos municipales a través de políticas, recursos y programas (Gran y Bernache, 2016). Aquí, cada gobierno local debe tener la capacidad de realzar la cadena de valor, donde está incluido el personal operativo, infraestructura y equipos, utilizados para la recolección y el manejo de residuos; en función de generar bienestar en la población y el cuidado del medio ambiente (Pasco, 2015). Por último, la responsabilidad social ambiental hace referencia al compromiso que deben demostrar no solo los ciudadanos, sino, también las empresas y quienes administran los gobiernos a nivel nacional, regional y local. En este caso, lo que se espera es que se compatibilice las actividades comerciales y coorporativas con la preservación del medio ambiente (Alcocer et al., 2019). Un concepto arraigado al tema del desarrollo sostenible, donde se espera que exista un equilibrio entre el crecimiento económico y el respeto por el medio ambiente (Abarca-Guerrero, 2015).

En suma, tomando en cuenta que en el departamento de Puno los residuos recolectados no son adecuadamente gestionados, siendo estos destinados al quemado o al incinerado en un $36.1 \%$, por encima de la región Lima que incinera un 30.2\%. Además, que Juliaca es la ciudad con mayores puntos críticos de contaminación ambiental en la región (Instituto Nacional de Estadística e Informatica, 2019), el objetivo de la presente investigación es determinar los factores asociados que explican el cumplimiento de la normatividad de la gestión Integral de Residuos Sólidos en la municipalidad de San Román, Juliaca.

\section{Metodología}

\section{Diseño}

Este estudio corresponde a un estudio de enfoque cuantitativo, de tipo explicativo, diseño no experimental y corte transversal (Hernández et al., 2014). Bajo un muestreo no probabilístico intencional, se contó con la participación voluntaria de 96 trabajadores de la municipalidad de San Román-Juliaca, del área de Gestión Ambiental: personal administrativo (32) y personal operativo (64). De estos, $54.7 \%$ son mujeres y $45.3 \%$ son varones, de entre 20 a 51 años de edad. 


\section{Instrumentos}

\section{Escala de cumplimiento de la normatividad de gestión integral de residuos sólidos}

Fue creada por los autores para fines de la presente investigación. Tiene el objetivo de evaluar el nivel de cumplimento de la Ley de Gestión Integral de Residuos Sólidos (RS) en los trabajadores administrativos. Está compuesto por 21 ítems con opciones de respuesta en formato tipo Likert: siempre (5), casi siempre (4), regularmente (3), algunas veces (2), nunca (1). Demostró validez basada en el contenido a través de la valoración por juicio de 5 expertos quienes evaluaron la relevancia, representatividad, y claridad de los ítems (V de Aiken >.70; IC 95\%: > .75 - .91); así también la confiabilidad en la presente investigación fue aceptable (Alpha de Cronbach [ $\alpha=.952]$ ).

\section{Cuestionario de cumplimiento de la normatividad de gestión integral de residuos sólidos}

Fue creada por los autores para fines de la presente investigación. Tiene el objetivo de evaluar el nivel de cumplimento de la Ley de Gestión Integral de Residuos Sólidos (RS) en los trabajadores operativos. Está compuesto por 17 ítems con opciones de respuesta dicotómica: si (2) y no (1). Demostró validez basada en el contenido a través de la valoración por juicio de 5 expertos quienes evaluaron la relevancia, representatividad, y claridad de los ítems (V de Aiken >.75; IC 95\%: > .79 - .95); así también la confiabilidad en la presente investigación fue aceptable (Alpha de Cronbach $[\alpha=.799])$.

Escala de factores asociados al cumplimiento de la normatividad de gestión integral de residuos sólidos

Creado por los autores para fines de la presente investigación. Tiene el objetivo de evaluar en que medida el presupuesto, gestión publica y responsabilidad social influyen sobre el cumplimento de la Ley de Gestión Integral de Residuos Sólidos, en trabajadores administrativos y operativos. Está compuesto por 14 ítems con opciones de respuesta en formato tipo Lickert: siempre (5), casi siempre (4), regularmente (3), algunas veces (2), nunca (1). Demostró validez basada en el contenido a través de la valoración por juicio de 5 expertos quienes evaluaron la relevancia, representatividad, y claridad de los ítems (V de Aiken >.70; IC 95\%: > .75 - .90); así también la confiabilidad en la presente investigación fue aceptable (Alpha de Cronbach $[\alpha=.943]$ ).

\section{Procedimientos}

Una vez obtenida la autorización por parte de la municipalidad de Juliaca, se recolectó los datos entre el 12 al 24 de junio del año 2020 a través de un personal administrativo de la oficina de 
gestión ambiental. En la primera parte de las encuestas se colocó el consentimiento informado donde se explicaba el objetivo y fines de la investigación, además, se enfatizó la declaración de que los datos brindados iban a ser manejados con total confidencialidad. Este fue explicado antes de que los participantes respondieran a las preguntas y reactivos.

\section{Análisis estadístico}

Para los análisis estadísticos, se utilizó el programa estadístico SPSS versión 25,0 y se realizó por etapas. En la primera etapa, se calculó las frecuencias y porcentajes de las variables demográficas y las variables de estudio. En la segunda etapa, se realizó un análisis de correlación de Pearson entre las variables y por último un análisis de regresión lineal multivariado.

\section{Resultados}

Según la tabla 1, el $68 \%$ del personal administrativo tenía entre 20 a 30 años de edad, mientras que el $39.1 \%$ de personal operativo tenía entre 31 a 40 años. Por otra parte, $65.6 \%$ del personal administrativo eran mujeres al igual que un $54.7 \%$ del personal operativo. Finalmente, $53.1 \%$ del personal administrativo tiene estudios a nivel superior, mientras que el $59.4 \%$ del personal operativo a nivel secundario.

\section{Tabla 1}

Características demográficas de los encuestados

\begin{tabular}{llcccc}
\hline \multirow{2}{*}{ Variables demográficas } & \multicolumn{2}{c}{ Categorías } & \multicolumn{2}{c}{ Personal administrativo } & \multicolumn{2}{c}{ Personal operativo } \\
& & $\mathrm{f}$ & $\%$ & $\mathrm{f}$ & $\%$ \\
\hline Edad & 20 a 30 años & 22 & 68.8 & 20 & 31.3 \\
& 31 a 40 años & 6 & 18.8 & 25 & 39.1 \\
& 41 a 50 años & 3 & 9.4 & 12 & 18.8 \\
& 51 años a más & 1 & 3.1 & 7 & 10.9 \\
& Total & 32 & 100.0 & 64 & 100.0 \\
Genero & Masculino & 21 & 65.6 & 29 & 45.3 \\
& Femenino & 11 & 34.4 & 35 & 54.7 \\
\multirow{5}{*}{ Grado de instrucción } & Total & 32 & 100.0 & 64 & 100.0 \\
& Primaria & 2 & 6.3 & 13 & 20.3 \\
& Secundaria & 6 & 18.8 & 38 & 59.4 \\
& Técnico & 7 & 21.9 & 13 & 20.3 \\
& Superior & 17 & 53.1 & 0 & 0.0 \\
& Total & 32 & 100.0 & 64 & 100.0 \\
\hline
\end{tabular}


En cuanto al cumplimiento de la Ley de Gestión Integral de Residuos Sólidos, en mayor proporción el $40.6 \%$ del personal administrativo de la oficina de gestión ambiental de la municipalidad de Juliaca auto percibe que el nivel de cumplimiento es deficiente y solo un $28.1 \%$ refiere que es óptimo. En cambio, el $67.2 \%$ del personal operativo refiere que el cumplimiento es regular y solo un $3.1 \%$ que es óptimo.

En caso de la gestión del presupuesto, $37.5 \%$ del personal administrativo refiere que la gestión en este ámbito es deficiente, mientras que solo un $28.1 \%$ afirma que es óptimo. De igual forma, el $35.9 \%$ del personal operativo considera que es deficiente y el $29.7 \%$ que es óptimo. Seguidamente, en caso de la gestión pública, 59.4\% auto percibe que la gestión en este aspecto es regular, mientras que un $9.4 \%$ refiere que es óptima. Por otra parte, $43.8 \%$ del personal operativo refiere que la gestión en este aspecto es deficiente y 15.6\% que es óptima. Finalmente, en cuanto a la responsabilidad social, $46.9 \%$ del personal administrativo auto percibe un nivel regular y $21.9 \%$ un nivel alto. Por otra parte, $62.5 \%$ considera un nivel de responsabilidad social bajo y el resto $(37.5 \%)$ regular, evidenciando que ninguno en este grupo considera la existencia a un nivel alto.

\section{Tabla 2}

Percepción del cumplimiento de la normatividad de gestión integral de residuos sólidos y evaluación de los factores asociados

\begin{tabular}{llcccc}
\hline \multirow{2}{*}{ Variables } & \multicolumn{1}{c}{ Nivel } & Personal administrativo & \multicolumn{2}{c}{ Personal operativo } \\
& & $\mathrm{f}$ & $\%$ & $\mathrm{f}$ & $\%$ \\
\hline Cumplimiento de la Ley GIRS & Deficiente & 13 & 40.6 & 19 & 29.7 \\
& Regular & 10 & 31.3 & 43 & 67.2 \\
& Optimo & 9 & 28.1 & 2 & 3.1 \\
& Total & 32 & 100.0 & 64 & 100.0 \\
Gestión del presupuesto & Deficiente & 12 & 37.5 & 23 & 35.9 \\
& Regular & 11 & 34.4 & 22 & 34.4 \\
& Optimo & 9 & 28.1 & 19 & 29.7 \\
Gestión pública & Total & 32 & 100.0 & 64 & 100.0 \\
& Deficiente & 10 & 31.3 & 28 & 43.8 \\
& Regular & 19 & 59.4 & 26 & 40.6 \\
Responsabilidad social & Optimo & 3 & 9.4 & 10 & 15.6 \\
& Total & 32 & 100.0 & 64 & 100.0 \\
& Bajo & 10 & 31.3 & 40 & 62.5 \\
& Regular & 15 & 46.9 & 24 & 37.5 \\
& Alto & 7 & 21.9 & 0 & 0.0 \\
& Total & 32 & 100.0 & 64 & 100.0 \\
\hline
\end{tabular}


La tabla 3 da cuenta de la existencia de correlaciones significativas entre las variables de estudio. En este caso, correlaciones directas y moderadas entre el cumplimiento y el factor gestión pública $(\mathrm{r}=.502, \mathrm{r}=.671 ; \mathrm{p}<.01)$, el factor gestión presupuestal $(\mathrm{r}=.827, \mathrm{r}=.593 ; \mathrm{p}<.01)$ y el factor responsabilidad social $(\mathrm{r}=.794, \mathrm{r}=.407 ; \mathrm{p}<.01)$.

\section{Tabla 3}

Análisis de correlación entre las variables de estudio

\begin{tabular}{lcccc}
\hline & \multicolumn{4}{c}{ Cumplimiento de la normatividad } \\
& Personal administrativo & \multicolumn{2}{c}{ Personal operativo } \\
& $\mathrm{R}$ & $\mathrm{p}$ & $\mathrm{r}$ & $\mathrm{p}$ \\
\hline Factor gestión publica & $.502 * *$ & .003 & $.671 * *$ & .000 \\
Factor gestión presupuestal & $.827 * *$ & .000 & $.593 * *$ & .000 \\
Factor responsabilidad social & $.794 * *$ & .000 & .407 & .001 \\
\hline
\end{tabular}

Nota: ** significancia al nivel .01.

Según la tabla 4, los resultados del análisis de regresión múltiple, muestran un ajuste adecuado del modelo (Prueba $\mathrm{F}$ en el personal administrativo $=11.787$ y personal operativo $=$ 21.869, $\mathrm{p}<.01$ ), donde la gestión del presupuesto (personal administrativo: $\beta=.53, \mathrm{p}<.01$; personal operativo: $\beta=.51, \mathrm{p}<.01$ ), la gestión pública (personal administrativo: $\beta=.78, \mathrm{p}<.01$; personal operativo: $\beta=.42, \mathrm{p}<.01$ ) y la responsabilidad social (personal administrativo: $\beta=.77$, $\mathrm{p}<.01$; personal operativo: $\beta=.42, \mathrm{p}<.01$ ) son variables que predicen significativamente el cumplimiento de la normatividad de la gestión integral de residuos sólidos ( $\mathrm{R}^{2}$ ajustado en el personal administrativo $=.25, .59, .59$; y en el personal operativo $=.24, .16, .16$ ). Asimismo, los valores t de los coeficientes de regresión beta de las variables predictoras son altamente significativas $(\mathrm{p}<.01)$.

Nota: Variable dependiente $=$ cumplimiento de la normatividad de la gestión integral de residuos sólidos (Prueba F en el personal administrativo $=11.787$, personal operativo $=21.869, \mathrm{p}<.01$ ), $\mathrm{EE}=$ Error estándar, $\beta=$ Coeficiente de regresión estandarizado. $* \mathrm{R}^{2}$ ajustado.

\section{Tabla 4}


Factores que explican el cumplimiento de la normatividad de la gestión integral de residuos en la municipalidad de Juliaca

\begin{tabular}{lcccccccccc}
\hline \multirow{2}{*}{ Modelo } & \multicolumn{4}{c}{ Personal operativo } & \multicolumn{4}{c}{ Personal operativo } \\
\cline { 2 - 11 } & $\mathrm{R}^{2^{*}}$ & $\mathrm{EE}$ & $\beta$ & $\mathrm{t}$ & $\mathrm{Sig}$ & $\mathrm{R}^{2 *}$ & $\mathrm{EE}$ & $\beta$ & $\mathrm{t}$ & $\mathrm{Sig}$ \\
\hline 1(Constante) & & 10.95 & & 2.85 & .002 & & 2.30 & & 5.44 & .000 \\
Gestión del presupuesto & .258 & .52 & .53 & 3.43 & .002 & .249 & .13 & .51 & 4.67 & .000 \\
Gestión publica & .598 & .41 & .78 & 6.86 & .000 & .163 & .14 & .42 & 3.64 & .001 \\
Responsabilidad social & .592 & .38 & .77 & 6.78 & .000 & .165 & .12 & .42 & 3.67 & .001 \\
\hline Nota: *coeficiente de determinación ajustado. & & & & & & & &
\end{tabular}

\section{Discusión}

La gestión de residuos sólidos ha demostrado ser todo un desafío en el Perú, específicamente en la ciudad de Juliaca, donde en los últimos años se ha evidenciado dificultades en la gobernabilidad, conflictos sociales, así como repercusiones en la salud de la población (Huamaní, Tudela \& Huamaní, 2020). Ante ello la presente investigación tuvo el objetivo de determinar los factores asociados que explican el cumplimiento de la normatividad de la gestión Integral de Residuos Sólidos en la municipalidad de San Román, Juliaca.

Respecto al factor gestión de presupuesto y su relación explicativa sobre el cumplimiento de la Ley Integral de Residuos Sólidos, los resultados encontrados indican que este explica en un $25.8 \%$ y $24.9 \%$ (área administrativa y operativa) la varianza de la variable cumplimiento de la Ley GIRS, indicando que si se mejora la gestión del presupuesto el nivel de cumplimiento también mejorará. Estos resultados corroboran relativamente lo hallado por Tumi y Escobar (2018) quienes investigaron la incidencia de factores sociales y políticos en la inversión ambiental del Gobierno Regional de Puno - Perú, hallando que las inversiones en proyectos estratégicos ambientales son mínimos y poco significativos, además, en el análisis que realizaron entre los ejercicios 2015 al 2018 hallaron que, del total de proyectos ejecutados, menos del 50\% corresponde a proyectos ambientales. Al respecto, una explicación teórica ante estos hechos surge al reconocer que los gobiernos locales priorizan aspectos sociales y políticos antes que el aspecto ambiental (Pichardo, 2009). Esta realidad es latente en la municipalidad a pesar de que es uno de las únicas provincias que cuentan con plan de gestión ambiental, tanto el área administrativa como la operativa solo consideran un manejo óptimo en la gestión del presupuesto en $28.1 \%$ y $29 \%$ respectivamente.

Respecto al factor gestión pública y su poder explicativo sobre el cumplimiento de la Ley de gestión Integral de Residuos Sólidos, los resultados indican que este factor explica en un 59.8\% 
y $16.3 \%$ (área administrativa y operativa) la varianza de la variable cumplimiento de la Ley GIRS. Esto corrobora lo hallado por Rentería y Zeballos (2014) quienes a través de su estudio realizaron una propuesta de mejora para la gestión estratégica del programa de segregación en la fuente y recolección selectiva de residuos sólidos domiciliarios en el distrito de Los Olivos, obteniendo como resultados cuatro factores problemáticos como son el bajo nivel de organización y planificación, las escasas estrategias de capacitación al personal y sensibilización a la población y un bajo nivel de negociación para realizar convenios estratégicos. Esta situación implica la importancia de implementar estrategias eficientes de gestión como medio para acatar los planes y objetivos establecidos en el territorio nacional (Gonzales, 2015); por ello es preciso mejorar la gestión pública para mejorar el nivel de cumplimiento de la ley vigente, toda vez que solo califican de óptima la gestión un 9.4\% desde el área administrativa y un 15.6\% desde el área operativa.

Respecto al factor responsabilidad social y su poder explicativo sobre el cumplimiento de la Ley de Gestión Integral de Residuos Sólidos, los resultados indican que este factor explica en un $59.2 \%$ y $16.5 \%$ (área administrativa y operativa) la varianza de la variable cumplimiento de la Ley GIRS. Estos resultados confirman los hallazgos de Ayazo, Patiño y Pion (2014) quienes a través de su investigación titulada "Responsabilidad social para el manejo de residuos urbanos en el municipio de Turbana - Bolívar, Caribe Colombiano", confirmaron que el cumplimiento de las responsabilidades legales de los diferentes actores sociales (estado y comunidad) en lo referente al manejo adecuado de los residuos urbanos ha sido nula. Estos hechos se explican en función de reconocer la importancia de que todo servidor público debe tener en cuenta la responsabilidad social dentro de una gestión; para que esta sea más consciente y ecuánime dentro sistema económico global; por ello, es necesario estimular la responsabilidad social y conciencia ambiental (Cuevas y Rodríguez, 2016). De allí que es preciso que la responsabilidad social sea promovida por el estado y las municipalidades, ya que el estudio refleja que solo se califica como alto el nivel de responsabilidad social desde el área administrativa en un 21,9\%; sin embargo, desde el área operativa que más cercana está la población y conoce el reflejo de una cultura responsable ambientalmente, califican en un nivel malo en un 62,5\% y califican como óptima el cumplimiento de la ley en un $3,1 \%$.

Los resultados hasta aquí discutidos también guardan relación y corroboran las deficiencias mostradas en el informe de auditoría 097-2019 CG/GRPU-VP realizado por la Contraloría General de la Republica (2019), a la Municipalidad Provincial de San Román. Por ejemplo, el informe 
mencionado revela la existencia de acumulación de residuos sólidos en espacios públicos y laderas de ríos, ausencia de estrategias de difusión para que la población conozca el horario de recolección de la basura y ausencia de supervisión técnica durante la recolección, esto, claramente evidencia deficiencias en la gestión municipal, que precisamente es percibida como regular por el 59,4\% de trabajadores administrativos y deficiente según la percepción del 43,8\% de trabadores operativos. La presente investigación demuestra algunas limitaciones. Primero, la cantidad de la muestra y unidades de análisis que corresponden a una sola municipalidad. Al respecto, en el departamento de Puno existen otras 12 provincias e incluso, existen otros departamentos en el Perú con problemática similar a la estudiada, no obstante, en el contexto de la emergencia sanitaria fue complicado realizar gestiones para conseguir más participantes. Segundo, el método de recolección de datos fue la encuesta, es decir medidas de autorreporte. Aunque hubiera sido favorable realizar entrevistas, las restricciones a causa de la pandemia COVID-19 limitaron el uso de este recurso. Ante ello, se recomienda que futuras investigaciones incluyan este método de recolección de datos y busquen ampliar el espectro de la muestra.

\section{Conclusión}

Este trabajo ha expuesto que la calidad de la gestión pública, gestión presupuestal y responsabilidad social, son factores que explican el cumplimiento de la normatividad de gestión de residuos sólidos municipales, según la percepción de los trabajadores administrativos y operativos de la oficina de gestiona ambiental de la municipalidad de Juliaca. Estos hallazgos se suman a los dispuestos en la literatura científica peruana e internacional en cuestión de gestión de residuos sólidos; además, tiene repercusiones en el campo de la auditoria y gestión ambiental, específicamente la labor del auditor dentro del contexto del cuidado del medio ambiente.

\section{Referencias}

Abarca, L., Maas, G., \& Hogland, W. (2015). Desafíos en la gestión de residuos sólidos para las ciudades de países en desarrollo. Revista Tecnología En Marcha, 28 (2), 141. https://www.scielo.sa.cr/pdf/tem/v28n2/0379-3982-tem-28-02-00141.pdf

Abarca, D., Gutierrez, S., Escobar, F., \& Huata, P. (2018). Manejo de residuos sanitarios: un programa educativo del conocimiento a la práctica. Journal of High Andean Research, 20 (3), 315-324. http://www.scielo.org.pe/pdf/ria/v20n3/a05v20n3.pdf

Abelino, G., Quispe-Limaylla, A. G., Pérez, L. M., Leos-Rodríguez, J. A., Carranza, O., \& Flores, 
D. (2019). Factores asociados con la participación de las familias en la separación de residuos sólidos urbanos en Texcoco, Estado de México. Acta Universitaria, 29, 1-12. http://www.scielo.org.mx/pdf/au/v29/2007-9621-au-29-e2087.pdf

Alcocer, P., Cevallos, O., \& Knudsen, J. (2019). Mejoramiento de la gestión integral de los residuos solidos urbanos en el Cantón de Quevedo, Ecuador. Universidad y Sociedad, 11 (5), 362-367. http://scielo.sld.cu/pdf/rus/v11n5/2218-3620-rus-11-05-362.pdf

Ayazo, M., Patiño, A., \& Pion, A. (2014). Responsabilidad social para el manejo de residuos sólidos urbanos en el Municipio de Turbana-Bolivar, Caribe Colombiano (Tesis de posgrado, Pontificia Universidad Javeriana, Cartagena de Indias, Colombia). https://repository.javeriana.edu.co/bitstream/handle/10554/16849/AyazoMontoyaMonica20 14.pdf? sequence $=1 \&$ is Allowed $=\mathrm{y}$

Banco Mundial (2018). Los desechos: un análisis actualizado del futuro de la gestión de los desechos sólidos. http://bit.ly/3rvArrF

Bustios, C., Martina, M., \& Arroyo, R. (2013). Deterioro de la calidad ambiental y la salud en el Perú actual. Revista Peruana de Epidemiología, 17 (1), 1-9. https://www.redalyc.org/pdf/2031/203128542001.pdf

Cuevas, R. \& Rodríguez, R. (2016). Responsabilidad social y ética profesional en la gestión de la administración pública y empresarial. Revista Pensamiento y Gestión, (42), 1-25. http://www.scielo.org.co/pdf/pege/n42/2145-941X-pege-42-00006.pdf

Contraloría General de la República (2019). Informe de visita preventiva N097-2019-CG/L455$V P$. https://s3.amazonaws.com/spic-informespublicados/informes/2019/04/2019CSIL45500053_ADJUNTO.pdf

Diario Gestión. (2014). Oefa: Solo 17 gobiernos regionales planifican acciones de fiscalización ambiental. http://bit.ly/39XpGZ1

Diario Oficial El Peruano. (2017). Decreto Legislativo que prueba la Ley de Gestión Integral de Residuos Sólidos D.L. $N^{\circ}$ 1278. https://bit.ly/3tw4ce1

Gonzales, R. (2015). Implementación de la estrategia de desarrollo municipal, eje articulador del sistema de gestión del desarrollo local. Revista Retos de la Dirección, 9 (1), 121-144. http://scielo.sld.cu/pdf/rdir/v9n1/rdir06115.pdf

Gran, J. A., \& Bernache, G. (2016). Gestión de residuos sólidos urbanos, capacidades del gobierno municipal y derechos ambientales. Sociedad y Ambiente, 1 (9), 73-101. https://www.redalyc.org/pdf/4557/455745080004.pdf

Huamaní. C., Tudela. J., \& Huamaní. A. (2020). Gestión de residuos sólidos de la ciudad de Juliaca - Puno -Perú. Revista de Investigaciones Altoandinas, 22 (1), 49-56. http://www.scielo.org.pe/pdf/ria/v22n1/2313-2957-ria-22-01-106.pdf

Infobae. (2017). El atlas de los desperdicios: los países que más basura producen. 
http://bit.ly/2LtQqax

Instituto Nacional de Estadística e Informatica. (2019). Perú: Anuario de Estadisticas Ambientales. https://bit.ly/2Ltcmm1

Ministerio de Economia y Finanzas (2019). Implementación de un sistema integrado de manejo de residuos solidos municipales. https://bit.ly/2MJHgHl

Ministerio de Economia y Finanzas (2020). Programa presupuestal 0036: Gestión integral de residuos solidos. https://www.mef.gob.pe/contenidos/presu_publ/ppr/prog_presupuestal/articulados/articulad o_0036_2019.pdf

Oldenhage, F. (2016). Propuesta de un programa de gestión para mejorar el manejo de los residuos sólidos en el distrito de San Juan de Miraflores. Industrial Data, 19 (2), 07. https://www.redalyc.org/pdf/816/81649428002.pdf

Pasco, J. (2015). La modernización del estado en el Perú. Revista de Estudio de Políticas Públicas, 1 246-252. https://revistaestudiospoliticaspublicas.uchile.cl/index.php/REPP/article/view/38487

Pichardo, I. (2009). Responsabilidades municipales en materia ambiental. Revista Convergencia, 16 (49), 291-398. http://www.scielo.org.mx/pdf/conver/v16n49/v16n49a12.pdf

Quillos, S. A., Escalante, N. J., Sánchez, D. A., Quevedo, L. G., \& De La Cruz, R. A. (2018). Residuos Sólidos Domiciliarios: Caracterización Y Estimación Energética Para La Ciudad De Chimbote. Revista de La Sociedad Química Del Perú, 84 (3), 322-335. http://www.scielo.org.pe/pdf/rsqp/v84n3/a06v84n3.pdf

Rentería, J., \& Zeballos, M. (2014). Propuesta de mejora para la gestión estratégica del programa de segregación en la fuente y recolección selectiva de residuos sólidos domiciliarios en el distrito de Los Olivos. (Tesis de licenciatura, Pontificia Universidad Católica del Perú, Lima). http://tesis.pucp.edu.pe/repositorio/bitstream/handle/20.500.12404/6285/RENTERIA JOSE ZZEBALLOS_MARIA_PROPUESTA_MEJORA.pdf?sequence=1\&isAllowed=y

Salazar-Acuña, E. (2020). Indicador económico para la evaluación de la gestión municipal de los residuos valorizables en Costa Rica. Revista de Ciencias Ambientales, 54 (1), 1-15. Recuperado de: https://www.scielo.sa.cr/pdf/rca/v54n1/2215-3896-rca-54-01-1.pdf

Sánchez-Muñoz, M. del P., Cruz-Cerón, J. G., \& Maldonado-Espinel, P. C. (2019). Gestión de residuos sólidos urbanos en América Latina: un análisis desde la perspectiva de la generación. Revista Finanzas y Política Económica, $11 \quad$ (2), 321-336. http://www.scielo.org.co/pdf/fype/v11n2/2248-6046-fype-11-02-321.pdf

Tumi, J. E., \& Escobar-Mamani, F. (2018). Incidencia de factores sociales y políticos en la inversión ambiental del Gobierno Regional de Puno - Perú. Revista de Investigaciones Altoandinas, 20 (2), 235-250. http://www.scielo.org.pe/pdf/ria/v20n2/a08v20n2.pdf 